\title{
Oxygen Uptake and Energy Expenditure during Treadmill Walking with Masai Barefoot Technology (MBT) Shoes
}

\author{
TerJe F. GJøVAag, PhD ${ }^{1)}$, Ingar DAhlen, PT ${ }^{1)}$, Hå KON SANDVIK, PT ${ }^{1)}$, \\ PEYMAN MIRTAHERI, PhD ${ }^{2}$ \\ 1) Physiotherapy programme, Faculty of Health Sciences, Oslo University College: Post box 4, St Olav's \\ Plass, N-0130 Oslo, Norway. TEL: +47 22452474, FAX: +47 22452490, \\ E-mail: terje.gjovaag@hf.hio.no \\ ${ }^{2)}$ Faculty of Engineering, Oslo University College
}

\begin{abstract}
Purpose] It is suggested that walking with Masai Barefoot Technology (MBT) shoes will increase oxygen uptake and result in greater consumption of energy relative to walking with conventional shoes, but this has not been sufficiently investigated. [Methods] To examine this supposition, ten subjects walked on a treadmill with different types of shoes (jogging or MBT shoes), treadmill inclinations (zero or $10 \%$ inclination) and walking speeds (i.e. selfselected walking speed or fast walking speed). Oxygen uptake, heart rate, lung ventilation, ratings of perceived exertion and energy expenditure were measured during all walking conditions by a stationary metabolic cart. [Results] On a flat treadmill at self-selected and fast walking speeds, physiological responses were similar for jogging and MBT shoes. In contrast, fast uphill walking with MBT shoes significantly increased oxygen uptake by about $5 \%$ relative to jogging shoes. The calculated energy expenditure for 60 minutes of fast, uphill walking was about $6 \%$ higher when using MBT shoes. [Conclusion] The magnitude of increases in oxygen uptake and energy expenditure when using MBT shoes were quite small, and the clinical relevance regarding reductions in body weight may be negligible.
\end{abstract}

Key words: Walking speed, Inclination, Unstable shoes

(This article was submitted Aug. 11, 2010, and was accepted Aug. 27, 2010)

\section{INTRODUCTION}

Conventional shoes for walking and running are usually constructed to provide stability for the users. In contrast, Masai Barefoot Technologies (MBT, Switzerland) has developed a shoe that has a soft midsole and a rounded outsole in the anterior-posterior direction.

Consequently, the MBT shoe provides an "unstable" base compared to more conventional shoes. MBT shoes are widely used in rehabilitation medicine for various therapeutic and ergonomic purposes, and it has been suggested that using MBT shoes may reduce pain during walking for osteoarthritis patients ${ }^{1}$, improve the balance of disabled children ${ }^{2)}$ and prevent injuries in athletes ${ }^{3)}$. In addition, the manufacturer of MBT shoes claims (e.g. http:/ /us.mbt.com/Home/Benefits.aspx, accessed August 9, 2010) that using MBT shoes increases oxygen uptake both when standing still and during locomotion. They also suggest that using unstable MBT shoes burns more calories compared to more stable shoes. In this regard, Romkes et al. ${ }^{4}$ point out that obese people use MBT shoes during walking to enhance energy expenditure and reduce body fat. Despite these statements, we are not aware of any publications that have demonstrated that the use of MBT shoes does in fact increase energy expenditure relative to conventional jogging shoes. Because of the widespread use of MBT shoes in clinical settings, it is important to investigate if using MBT shoes affects oxygen uptake and energy expenditure during walking at different speeds and inclinations on a motorized treadmill.

The purpose of this study was therefore to investigate the following hypotheses:

H1. Inexperienced users of MBT shoes have a higher oxygen uptake when standing still compared to when wearing conventional jogging shoes

$H 2$. Oxygen uptake and energy expenditure is higher when walking with MBT shoes on a flat treadmill compared to walking with jogging shoes.

H3. Oxygen uptake and energy expenditure is higher when walking with MBT shoes on a treadmill with a $10 \%$ incline compared to walking with jogging shoes.

\section{SUBJECTS AND METHODS}

\section{Subjects}

Ten subjects volunteered for this study, five females and 
five males. The subjects were all fit, physically active, nonsmoking and healthy and were not using medication of any kind. The average (SD) age, height, weight and BMI of the subjects were 22.9 (1.5) years, $171.1(6.9) \mathrm{cm}, 66.1$ (10.5) $\mathrm{kg}$ and 22.5 (2.8), respectively. None of the participants had used the MBT shoe before and they did not have any musculoskeletal diseases or other conditions limiting their functional mobility. Written informed consent was obtained from all subjects. This study was approved by the Regional Committee for Medical Research Ethics in Norway.

\section{Methods}

The present study investigated the physiological responses to walking on a motorized treadmill with eight different experimental (EXP) protocols (Table 1). The experimental protocols varied by the type of shoe the subjects wore (jogging or MBT shoes), the treadmill inclination (zero or $10 \%$ inclination) and the walking speed (self-selected or fast walking speed). Zero inclination on the treadmill was the control situation, while ten percent inclination was chosen to expose the subjects to a physically more demanding walking situation that would increase muscle activation in the major muscles of the lower limbs ${ }^{10)}$.

Shoe conditions: The control shoes used in this study were a conventional type of running shoe in common use by the general population and with a typical "stable" midsole. The mean weight (SD) of the control shoes was 618 (67) grams (one pair). The unstable shoe tested in this study was the MBT Sports Black model from Masai Barefoot Technology (MBT, Switzerland). This shoe has a soft midsole and a rounded outsole in the anterior-posterior direction. Consequently, the MBT shoe provides an "unstable" shoe construction compared to the more stable control shoes. The mean weight (SD) of the MBT shoes was 1050 (75) grams (one pair).

Measurement equipment: Oxygen uptake $\left(\mathrm{VO}_{2}\right)$, lung ventilation (VE) and respiratory exchange ratio (RER) were measured by a stationary ergo spirometer (Sensor Medics Vmax229, CA, USA). Lactate (LA) levels in mixed venous blood were measured in the rest intervals by a Lactate Pro analyzer (Arkray, KDK Corporation, Shiga, Japan). Heart rate (HR) was monitored by a Polar heart rate monitor (Polar Electro, Kempele, Finland) and the Borg CR10 Scale was utilized to investigate the subjects' rating of perceived exertion (RPE) during the experiments ${ }^{5)}$. For all experimental protocols, we used a calibrated Woodway ELG70 motorized treadmill (Woodway, Weil am Rhein, Germany).

Measurements during standing still: Oxygen uptake during standing still with either jogging shoes or MBT shoes was determined during a period of 2 minutes for each shoe type. The sequence of this testing was randomized. The subjects were instructed to stand with parallel feet on the treadmill with equal weight distribution on the feet. To keep their balance, the subjects looked straight ahead. $\mathrm{VO}_{2}$ was reported every 20 seconds and averaged.

Measurements during treadmill testing: On day 1 of the experiments, the subjects reported to the laboratory after eating a standardized breakfast, rested for 30 minutes and
Table 1. The different experimental protocols

\begin{tabular}{cccc}
\hline $\begin{array}{l}\text { Experimental } \\
\text { Protocol }\end{array}$ & $\begin{array}{c}\text { Treadmill } \\
\text { inclination, } \%\end{array}$ & $\begin{array}{c}\text { Walking } \\
\text { Speed }\end{array}$ & $\begin{array}{c}\text { Type of } \\
\text { Shoe }\end{array}$ \\
\hline 1 & 0 & SSWS & JOGG \\
2 & 0 & SSWS & MBT \\
3 & 0 & FWS & JOGG \\
4 & 0 & FWS & MBT \\
5 & 10 & SSWS & JOGG \\
6 & 10 & SSWS & MBT \\
7 & 10 & FWS & JOGG \\
8 & 10 & FWS & MBT \\
\hline
\end{tabular}

SSWS $=$ self-selected walking speed, FWS $=$ fast walking speed, JOGG $=$ jogging shoes. MBT $=$ Masai Barefoot Technology shoes. The different experimental protocols were carried out in a random order.

then continued to determine their SSWS on the treadmill according to the protocol of Holt et $\mathrm{al}^{6}{ }^{6}$. The subjects adopted this walking speed also during walking with MBT shoes. The fast walking speed (FWS) was set by the researchers and was identical for all subjects and for both shoe types. Immediately following the determination of SSWS, the subjects performed an incremental running test on the treadmill to measure their maximal oxygen uptake. During $\mathrm{VO}_{2}$ max testing the subjects wore jogging shoes (JOGG). Following the $\mathrm{VO}_{2}$ max test, the subjects rested for about 24 hours before the different EXP protocols were conducted. The subjects were instructed to avoid physical activity and alcohol during this resting period.

On day 2 of the experiments, the subjects reported to the laboratory after eating a standardized breakfast and after a 30 minutes rest period, they subsequently performed the standing still test, followed by the eight different EXP sessions (Table 1) in a random order. Each EXP session lasted for a total of 10 minutes. Ventilatory and respiratory data during the last three minutes of each EXP session was reported every $20 \mathrm{sec}$., and averaged. Between each EXP session, the subjects rested for a minimum of 30 minutes. HR, LA and RPE were monitored during each rest period to ensure that the subjects were completely recovered before they started the next EXP session.

Measurements of step frequency and step length: Step frequencies were measured using a videotape recorder during treadmill walking sessions at each speed and inclination. Stride length was calculated by measurements of the distance and step frequency during the walking sessions.

Statistics: Q-Q plots showed that the data were normally distributed, hence parametric statistics were used. The data were analyzed using SPSS version 17.0. Group comparisons were performed using Student's t-test with a significance level of $p \leq 0.05$. The results are presented as means and standard deviations (SD).

\section{RESULTS}

Mean (SD) maximal values of $\mathrm{VO}_{2}, \mathrm{VE}, \mathrm{HR}, \mathrm{LA}, \mathrm{RER}$ and RPE following treadmill testing on day 1 were: 55.9 
Table 2. Physiological responses to treadmill walking with different types of shoes

\begin{tabular}{ccccccccc}
\hline $\begin{array}{c}\text { Treadmill } \\
\text { inclin. } \%\end{array}$ & $\begin{array}{c}\text { Walking } \\
\text { speed }\end{array}$ & $\begin{array}{c}\text { Shoe } \\
\text { type }\end{array}$ & $\begin{array}{c}\mathrm{VO}_{2} \mathrm{max}, \\
\text { percent }\end{array}$ & $\begin{array}{c}\mathrm{VO} 2, \\
\mathrm{~m} \mathrm{~kg}^{-1} \mathrm{~min}^{-1}\end{array}$ & $\begin{array}{c}\mathrm{VE}, \\
\mathrm{L} \mathrm{min}^{-1}\end{array}$ & $\begin{array}{c}\mathrm{HR}, \\
\text { beats } \mathrm{min}^{-1}\end{array}$ & $\begin{array}{c}\mathrm{RPE}, \\
(0-10)\end{array}$ & RER \\
\hline 0 & SSWS & JOGG & $20.6(1.8)$ & $11.4(1.2)$ & $17(3)$ & $88(9)$ & $1.0(0.5)$ & $0.85(0.1)$ \\
0 & SSWS & MBT & $21.1(2.5)$ & $11.7(1.3)$ & $19(4)$ & $88(9)$ & $1.2(0.5)$ & $0.87(0.1)$ \\
0 & FWS & JOGG & $27.3(3.2)$ & $15.1(0.8)$ & $23(4)$ & $104(15)$ & $1.7(0.7)$ & $0.87(0.1)$ \\
0 & FWS & MBT & $27.7(3.3)$ & $15.3(1.2)$ & $24(3)$ & $104(15)$ & $2.0(1.0)$ & $0.89(0.05)$ \\
10 & SSWS & JOGG & $41.1(4.9)$ & $22.8(1.9)$ & $31(4)$ & $120(12)$ & $2.4(0.9)$ & $0.88(0.05)$ \\
10 & SSWS & MBT & $41.5(5.8)$ & $22.9(2.8)$ & $31(4)$ & $121(13)$ & $2.5(0.8)$ & $0.88(0.03)$ \\
10 & FWS & JOGG & $52.5(6.7)$ & $29.0(1.7)$ & $38(8)$ & $137(14)$ & $3.6(1.2)$ & $0.90(0.03)$ \\
10 & FWS & MBT & $55.1(6.0)^{* *}$ & $30.5(1.4) * *$ & $44(7)^{* * *}$ & $146(18)^{*}$ & $4.5(1.7)^{*}$ & $0.92(0.03)^{*}$ \\
\hline
\end{tabular}

*** $\mathrm{p}<0.001, * * \mathrm{p}<0.01,{ }^{*} \mathrm{p}<0.05 ; 10 \%$ FWS MBT vs. $10 \%$ FWS JOGG.

Table 3. Time-distance parameters

\begin{tabular}{cccccc}
\hline $\begin{array}{c}\text { Treadmill } \\
\text { inclination, } \%\end{array}$ & $\begin{array}{c}\text { Walking } \\
\text { speed }\end{array}$ & $\begin{array}{c}\text { Type of } \\
\text { Shoe }\end{array}$ & $\begin{array}{c}\text { Cadence, } \\
\text { steps } \text { min }^{-1}\end{array}$ & $\begin{array}{c}\text { Step length, } \\
\text { m step }^{-1}\end{array}$ & $\begin{array}{c}\text { Stride length, } \\
\text { m stride }^{-1}\end{array}$ \\
\hline 0 & SSWS & JOGG & $110.2(0.8)$ & $0.68(0.06)$ & $1.36(0.12)$ \\
0 & SSWS & MBT & $109.8(2.7)$ & $0.68(0.06)$ & $1.36(0.12)$ \\
0 & FWS & JOGG & $121.8(2.9)$ & $0.78(0.02)$ & $1.58(0.03)$ \\
0 & FWS & MBT & $121.9(2.4)$ & $0.79(0.15)$ & $1.57(0.03)$ \\
10 & SSWS & JOGG & $103.8(2.9)$ & $0.72(0.06)$ & $1.43(0.10)$ \\
10 & SSWS & MBT & $104.7(2.8)$ & $0.71(0.06)$ & $1.42(0.12)$ \\
10 & FWS & JOGG & $119.2(3.3)$ & $0.80(0.02)$ & $1.61(0.04)$ \\
10 & FWS & MBT & $121.9(2.7)^{* * *}$ & $0.78(0.16)^{* * *}$ & $1.57(0.03)^{* * *}$ \\
\hline
\end{tabular}

Values are means and SD. ${ }^{* * *} \mathrm{p}<0.001 ; 10 \%$ FWS MBT vs. $10 \%$ FWS JOGG.

(6.7) $\mathrm{ml} \mathrm{kg}^{-1} \mathrm{~min}^{-1}, 114$ (31) $\mathrm{L} \mathrm{min}^{-1}, 194$ (6) beats $\mathrm{min}^{-1}$, $11.7(1.8) \mathrm{mmol} \mathrm{L}^{-1}, 1.15(0.02)$ and $8.7(0.8)$, respectively.

Oxygen uptake during standing still with MBT or jogging shoes were $4.4(1.0)$ and $4.4(0.7) \mathrm{ml} \mathrm{kg}^{-1} \mathrm{~min}^{-1}$, respectively. The oxygen uptake during the different EXP sessions increased in response to increasing walking speed and inclination of the treadmill (Table 2).

In general, the responses were similar for jogging shoes and MBT shoes, and $\mathrm{VO}_{2}$ increased from about $11 \mathrm{ml} \mathrm{kg}^{-1}$ $\min ^{-1}$ (about $20 \%$ of $\mathrm{VO}_{2} \max$ ) during level walking at SSWS, to about $30 \mathrm{ml} \mathrm{kg}^{-1} \mathrm{~min}^{-1}$ during FWS and $10 \%$ incline (about $50 \%$ of $\mathrm{VO}_{2} \max$ ). However, when the subjects were walking with FWS and $10 \%$ inclination on the treadmill, type of shoe had a significant influence on the physiological parameters (Table 2). In this situation, and relative to JOGG, $\mathrm{VO}_{2}$ increased $5.2 \%(\mathrm{p}<0.01)$, VE $15.8 \%$ $(\mathrm{p}<0.001)$, HR $6.6 \%(\mathrm{p}<0.05)$, RPE $25 \%(\mathrm{p}<0.05)$ and RER $2.2 \%(\mathrm{p}<0.05)$ when using MBT shoes.

Energy expenditure (kcal) was calculated using standard procedures and tables ${ }^{7}$ on the basis of measurements of oxygen uptake and RER values during steady state conditions. Given 60 minutes of physical activity, the mean (SD) energy expenditure during fast uphill walking with JOGG and MBT shoes was 567 (103) and 601 (102) kcal hour $^{-1},(\mathrm{p}<0.001)$, respectively. Hence, the difference in calorie consumption amounts to $34 \mathrm{kcal}^{\text {hour }}{ }^{-1}$ between these two shoe types.

Mean (SD) SSWS for the subjects was $1.24(0.1) \mathrm{m} \mathrm{sec}^{-1}$ $\left(74.4 \mathrm{~m} \mathrm{~min}^{-1}\right)$, while the fast walking speed (FWS) was set by the investigators to $1.60 \mathrm{~m} \mathrm{sec}^{-1}\left(96 \mathrm{~m} \mathrm{~min}^{-1}\right)$ for all subjects (about $30 \%$ faster than the SSWS), which is in the range reported for customary fast walking speeds of adults ${ }^{\gamma)}$.

These parameters were quite similar in the different experimental sessions regardless whether subjects were wearing MBT or jogging shoes (Table 3). However, compared to using jogging shoes, cadence increased by 2.3 $\%(\mathrm{p}<0.001)$, while step length decreased by $2.5 \%$ (both, $\mathrm{p}<0.001)$ when the subjects were wearing MBT shoes during FWS and $10 \%$ inclination on the treadmill.

$\mathrm{C}_{\mathrm{w}}\left(\mathrm{VO}_{2} \mathrm{ml} \mathrm{meter}^{-1}\right)$ during SSWS on a flat treadmill was $0.15(0.01)$ and $0.16(0.01)$ for JOGG and MBT shoes, respectively. At FWS, $\mathrm{C}_{\mathrm{w}}$ was $0.16(0.01)$ for both JOGG and MBT shoes.

$\mathrm{C}_{\mathrm{w}}$ during uphill walking with SSWS was 0.31 (0.02) for both JOGG and MBT shoes. $\mathrm{C}_{\mathrm{w}}$ during fast uphill walking with JOGG was $0.30(0.01)$ and $0.32(0.01)$ using MBT shoes $(\mathrm{p}<0.01)$.

\section{DISCUSSION}

Despite widespread use of MBT shoes in clinical settings, there have been few investigations of how walking with MBT shoes affects physiological responses under different walking conditions. The manufacturer of MBT shoes claims that using MBT shoes increases oxygen uptake and burns more calories compared to using more conventional shoes. Based on these claims, we have formulated several hypotheses $(H)$ in order to investigate whether MBT shoes affect oxygen uptake and energy expenditure under different walking conditions.

H1: We hypothesized that for inexperienced users of unstable MBT shoes, postural muscles would be activated to 
a greater extent during quiet standing compared to when wearing stable control shoes ${ }^{3}$. Consequently, oxygen uptake would be elevated. In the present study, however, different shoe types had no effect on the subjects' mean oxygen uptake $\left(4.4 \mathrm{ml} \mathrm{kg}^{-1} \mathrm{~min}^{-1}\right.$ for both JOGG and MBT shoes) during standing still. This indicates that minimal muscular activity is required for standing still and that wearing unstable shoes does not challenge postural control more than when using conventional jogging shoes.

Nigg et al. ${ }^{3)}$, however, observed increased electromyographic (EMG) activity in the $\mathrm{m}$. gastrocnemius of subjects when standing still in MBT shoes, compared to jogging shoes, but it is unknown if the increased EMG activity affected the subjects' oxygen uptake. An increased activation of a small muscle mass such as the $\mathrm{m}$. gastrocnemius, may, however, not be sufficient to stimulate to an increase in whole body oxygen uptake during standing still.

H2: For walking on a flat treadmill, we postulated that oxygen uptake would be higher using MBT shoes, relative to jogging shoes. During walking with SSWS on a flat treadmill the subjects in the present study used about $20 \%$ of their maximal aerobic capacity, regardless of type of shoe they were wearing. With FWS, this value increased to about $27 \%$ of $\mathrm{VO}_{2} \max$ (Table 2), but there were no differences in oxygen uptake between the stable and unstable shoe types. Interestingly, Nigg et al. ${ }^{9}$ measured oxygen uptake during slow running on a flat treadmill with shoes having either a soft, viscous (MBT like) midsole or a harder, elastic (jogging shoe like) midsole but found no difference in the mean oxygen uptake between these two shoe conditions. Collectively, there is little evidence supporting the claims of the MBT manufacturers that oxygen uptake increases during standing still and during slow running with MBT shoes.

H3: To our knowledge, this is the first study that has looked at physiological responses during uphill MBT walking. Initially, we hypothesized that walking with MBT shoes on a steep incline would increase oxygen uptake relative to jogging shoes. When the subjects walked at their SSWS there were, however, no significant differences in oxygen uptake or other physiological measurements between jogging and MBT shoes. Further analysis also showed that cadence and step length was similar for jogging shoes and MBT shoes, and consequently the gait pattern during uphill walking at SSWS was not affected by the type of walking shoe (Table 3). The situation, however, changed markedly during fast, uphill MBT walking.

During this walking session, oxygen uptake increased to about $55 \%$ of $\mathrm{VO}_{2} \mathrm{max}$, and $\mathrm{VO}_{2}$ was significantly higher when subjects wore MBT shoes. In contrast to SSWS, fast MBT walking resulted in an increased cadence and decreased step length compared to jogging shoes. MBT gait characteristics during fast, uphill walking were, however, not different from fast level walking. Consequently, we assume that the increased oxygen uptake during fast, uphill MBT walking is primarily related to the type of shoe being used and to the increase in treadmill inclination, and less to changes in gait characteristics per se.
The oxygen uptake during fast uphill walking was about $5 \%$ higher when subjects wore MBT shoes compared to jogging shoes, and one explanation for this may be differences in the magnitude of muscle activation during the walking sessions. In this respect, Lange et al. ${ }^{10)}$, observed that both average and peak EMG activity increased in the major muscles of the lower limb during treadmill walking with a $12 \%$ incline, compared to level treadmill walking. Regrettably, we have no data on EMG activity during uphill walking with jogging shoes and MBT shoes, but this will be pursued in a forthcoming study.

The MBT shoes weighed on average 432 (64) grams more than the control shoes, and one may question the impact of this increased mass on metabolic processes during this challenging walking condition. Accordingly, allometric scaling of mass allows a more dimensionless estimate of oxygen consumption. Regardless of allometric scaling, oxygen uptake with MBT shoes was still higher relative to

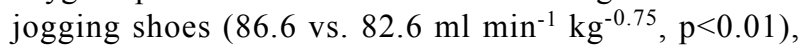
suggesting that the added inertia when MBT shoes were worn was not the principal mechanism for increased oxygen uptake during fast, uphill walking.

Finally, the manufacturer claims that using MBT shoes burns more calories both when standing still and during slow running. Because of this claim, it is no surprise that overweight people use MBT shoes in order to increase their energy expenditure and reduce body fat ${ }^{4}$. We calculated the energy expenditure during fast, uphill treadmill walking for both jogging shoes and MBT shoes. Given an exercise bout with a duration of 60 minutes, the total energy expenditure using jogging shoes is $567 \mathrm{kcal}^{\text {hour }}{ }^{-1}$, while the energy expenditure using MBT shoes is $601 \mathrm{kcal}^{-1}$ hour $^{-1}$. Thus, the mean difference in energy expenditure is $34 \mathrm{kcal} \mathrm{hour}^{-1}$, in favor of the MBT shoes. Hypothetically, if the subjects exercised for one hour every day for a whole year, the difference in calorie consumption would amount to about $12,000 \mathrm{kcal}$ per year. During fast uphill MBT walking, the RER was on average 0.92 , hence only $25 \%$ of the total energy consumption was derived from fat oxidation ${ }^{7)}$. Consequently, it is unlikely that the small increase in energy expenditure during fast, uphill MBT walking would reduce body fat to any great extent. In a clinical setting there may be other and faster methods of reducing bodyweight for obese and overweight people.

In conclusion, the present results show that walking in MBT shoes at normal walking speeds on a flat or inclined treadmill does not increase oxygen uptake compared to conventional jogging shoes. There is, however, increased oxygen uptake and energy expenditure during fast uphill walking when using MBT shoes. The difference in calorie consumption between jogging shoes and MBT shoes is probably too small to be of clinical relevance when it comes to weight regulation and reduction in body fat.

\section{ACKNOWLEDGEMENTS}

We thank Masai Norway for lending us the Masai shoes used in the present study. The company had no involvement in the study design, in collection, analysis and interpretation 
of data, in writing the manuscript or in the decision to submit this paper.

\section{REFERENCES}

1) Nigg BM, Emery C, Hiemstra LA: Unstable shoe construction and reduction of pain in osteoarthritis patients. Med Sci Sports Exerc, 2006, 38: 1701-1708.

2) Ramstrand N, Bjørk Andersson C, Rusaw D: Effects of an unstable shoe construction on standing balance in children with developmental disabilities: A pilot study. Prost Orthotics Int, 2008, 32: 422-433.

3) Nigg B, Hintzen S, Ferber R: Effect of an unstable shoe construction on lower extremity gait characteristics. Clin Biomech, 2006, 21, 82-88.

4) Romkes J, Rudmann C, Brunner R: Changes in gait and EMG when walking with the Masai Barefoot Technique. Clin Biomech, 2006, 21: 75-81.
5) Borg G: The Borg CR10 Scale: A method for measuring intensity of experience, e.g., perceived exertion and pain. Stockholm University. Stockholm, Sweden, 1998.

6) Holt KG, Hamill J, Andres RO: Predicting the minimal energy cost of human walking. Med Sci Sports Exerc, 1991, 23: 491-498.

7) Peronnet F, Massicotte D: Table of nonprotein respiratory quotient: An update. Can J Sport Sci, 1991, 16: 23-29.

8) Waters RL, Lunsford BR, Perry J et al.: Energy-Speed Relationship of Walking: Standard Tables. J Orthopaed Res, 1988, 6: 215-222.

9) Nigg BM, Stefanyshyn D, Cole G, et al.: The effect of material characteristics of shoe soles on muscle activation and energy aspects during running. J Biomech, 2003, 36: 569-575.

10) Lange GW, Hintermeister RA, Schlegel T, et al.: Electromyographic and kinematic analysis of graded treadmill walking and the implications for knee rehabilitation. J Orthopaed \& Sports Phys Ther, 1996, 23: 294-301. 